Les techniques numériques - Simulations 
Collection SFN 10 (2010) 427-447

(C) Owned by the authors, published by EDP Sciences, 2010

DOI: $10.1051 / \mathrm{sfn} / 2010007$

\title{
Diffusion inélastique des neutrons et simulations atomistiques
}

\author{
M.R. Johnson, M. Zbiri, M.A. González, E. Pellegrini, P. Calligari, L. Capogna, \\ E. Farhi, A. Filhol, R. Ghosh et D. Richard
}

Institut Laue Langevin, BP. 156, 6 rue Jules Horowitz, 38042 Grenoble, France

\begin{abstract}
Résumé. Des logiciels conviviaux et des ordinateurs multiprocesseurs d'un prix abordable sont maintenant disponibles et ouvrent aux expérimentateurs la porte du monde de la simulation pour "l'analyse avancée des données". La diffusion inélastique des neutrons est un partenaire idéal des simulations atomistiques car, comme ces dernières, elle explore les positions et le mouvement des atomes sur des échelles de longueur et de temps similaires. Du côté expérimental, la complexité croissante des systèmes étudiés (et donc de l'analyse des données) rend nécessaire l'utilisation de modèles de plus en plus élaborés et réalistes. Cet article décrit d'un point de vue pratique, les méthodes de simulation qui peuvent être utilisées pour interpréter les données de diffusion inélastique des neutrons, c'est-à-dire les techniques de dynamique des réseaux et de dynamique moléculaire. Les théories classiques à base de champs de force et de fonctionnelles de densité, toutes deux basées sur une connaissance des interactions interatomiques et de l'énergie totale, sont présentées ici comme les méthodes les plus adaptées.
\end{abstract}

\section{INTRODUCTION}

La diffusion inélastique des neutrons $(\mathrm{DIN})^{1}$ sonde la façon dont les atomes bougent et donne ainsi une signature expérimentale de cette dynamique. Selon les lois du mouvement de Newton, un atome se déplace parce qu'une force lui a été appliquée : il suit alors une trajectoire balistique. Dans la matière condensée, à cause de la forte densité en atomes et de leurs interactions mutuelles, ce régime balistique est de courte durée et les atomes subissent rapidement de nouvelles forces et accélérations. Pour un atome de masse $m$, l'accélération $\boldsymbol{a}$ dépend linéairement de la force $\boldsymbol{F}$ qui s'exerce sur lui :

$$
\boldsymbol{F}=m \boldsymbol{a} .
$$

Un ensemble d'atomes est dépourvu de mouvement quand ils sont tous dans des minima de la surface d'énergie potentielle $(\mathrm{SEP})^{2}$. Leur arrangement est alors une structure à l'équilibre. Plusieurs arrangements peuvent être envisageables. La structure la plus stable est celle dont l'énergie totale est la plus faible, c'est-à-dire celle qui correspond au minimum global de la SEP.

La structure à l'équilibre est le point de départ des calculs de dynamique des réseaux (DR ${ }^{3}$. Les mouvements de vibration mesurés par la DIN explorent la SEP au voisinage de ses minima. Etant donné que la SEP dépend de toutes les coordonnées atomiques, déplacer légèrement un seul atome de sa position d'équilibre induit une force de rappel sur cet atome et des forces interatomiques sur tous les autres atomes. Dans le cas d'un puits de potentiel harmonique, la valeur de la force de rappel dépend linéairement de l'amplitude du déplacement. Pour les systèmes réels, l'approximation harmonique n'est valable que pour de petits déplacements $(<0.05)$. Les forces interatomiques, évaluées en déplacant les

\footnotetext{
${ }^{1}$ En Anglais : INS pour "Inelastic Neutron Scattering".

2 En Anglais : PES pour "Potential Energy Surface".

3 En Anglais : LD pour "Lattice Dymamics".

This is an Open Access article distributed under the terms of the Creative Commons Attribution-Noncommercial License 3.0, which permits unrestricted use, distribution, and reproduction in any noncommercial medium, provided the original work is properly cited.
} 
atomes hors de leurs positions d'équilibre, sont utilisées pour construire la matrice dynamique (MD) ${ }^{4}$ qui est une expression des équations du mouvement de tous les atomes. La diagonalisation de la MD donne un ensemble de modes normaux $(\mathrm{MN})^{5}$, ou oscillateurs harmoniques; à chacun est associé une fréquence caractéristique et un vecteur de déplacement décrivant le mouvement relatif de tous les atomes impliqués. Les MN peuvent ensuite être utilisés pour calculer les grandeurs observables par diffusion de neutron (sur poudre ou monocristal), sous forme de densité généralisée d'états vibrationnels et de fonction de diffusion $\mathrm{S}(\mathrm{Q}, \omega)$. Dans le cas d'une structure à l'équilibre correspondant à un minimum de la SEP bien séparé des autres, les fréquences des modes normaux sont toutes supérieures ou égales à zéro. Toutefois un déplacement atomique plus important peut amener la structure vers un autre minimum de la SEP où l'atome déplacé vers sa position initiale n'a pas de force de rappel vers cette dernière. Dans ce cas, le calcul de DR donnera des fréquences négatives, ou plus exactement des fréquences imaginaires (voir plus loin), qui sont la signature de cette instabilité.

La structure à l'équilibre est aussi le point de départ des simulations de dynamique moléculaire $(\mathrm{DM})^{6}$. Dans une DM, les observables expérimentales de la DIN mentionnées ci-dessus sont déduites des trajectoires des atomes en fonction du temps via des fonctions de corrélation telles que la fonction d'auto-corrélation de vitesse (FACV) ${ }^{7}$ et la fonction de corrélation de van Hove. Pour cela il faut d'abord déplacer le système de sa position d'équilibre par une impulsion initiale après quoi le mouvement devient perpétuel. Une simulation de DM correspond donc à une série de structures, chaque nouvelle structure étant calculée à partir de la structure précédente en utilisant les forces instantanées et donc les accélérations qui s'exercent sur chacun des atomes.

Les forces sont donc centrales aux méthodes de DR et de DM. Elles sont calculées à partir d'une expression de l'énergie totale du système, comme étant les dérivées par rapport aux coordonnées atomiques appropriées. Il y a de nombreuses méthodes de calcul et d'approximation utilisées pour le calcul de l'énergie d'un système d'atomes, mais deux sont plus largement répandues et permettent d'aborder un grand nombre de systèmes et de domaines scientifiques.. Cette énergie dépend de la façon dont les électrons de valence des atomes s'organisent autour des électrons de coeur et des noyaux atomiques. Une description correcte de la structure électronique nécessite alors de résoudre l'équation de Schroedinger pour le système électrons - noyaux. Les méthodes dites de Hartree-Fock conduisent malheureusement à des volumes de calcul considérables pouvant être à l'échelle de la puissance septième du nombre d'atomes $\left(\mathrm{N}^{7}\right.$ avec $\mathrm{N}$ le nombre d'atomes), autrement dit doubler $N$ revient à un accroissement d'un facteur 128 du temps de calcul. Une des méthodes les plus efficaces est de calculer le champ de densité mono-électronique autour du noyau plutôt que la fonction d'onde multiélectronique. Cette approche est partie intégrante de la théorie des fonctionnelles de densité (TFD) ${ }^{8}$. Les volumes de calculs sont alors à l'échelle de $\mathrm{N}^{3}$. Avec les moyens de calculs actuels, les méthodes à base de TFD conviennent bien pour des systèmes périodiques comportant jusqu'à une centaine d'atomes et pour des échelles de temps atteignant 100 ps.

Des systèmes plus conséquents, comme les polymères et les bio-molécules, présentent, outre un nombre élevé d'atomes, des dynamiques plus lentes. L'échelle de temps à prendre en compte est donc plus importante, ce qui nécessite des méthodes de calcul dont le coût par atome est bien plus faible. On y parvient en traitant les électrons de façon implicite, en décrivant les liaisons chimiques formées par les électrons comme autant de ressorts. La densité électronique nette autour des atomes est décrite par des charges ponctuelles. La corrélation dynamique électronique à longue distance (non prise en compte dans les méthodes TFD actuelles) est décrite par le terme dispersif de l'interaction de Van der Waals (VDW), lequel est typiquement proportionnel à $r^{6}$ avec $r$ la distance interatomique. Dans ce contexte, on utilise

\footnotetext{
${ }^{4}$ En Anglais : DM pour "Dynamical Matrix".

5 En Anglais : NM pour Normal Modes.

6 En Anglais: MD pour "Molecular Dynamics".

7 En Anglais: VACF pour "Velocity Auto-Correlation Function".

${ }^{8}$ En Anglais : DFT pour "Density of Functional Theory".
} 
un champ de force $(\mathrm{CF})^{9}$ qui est une description analytique et paramétrique de l'énergie du système à base de ressorts, charges ponctuelles, interactions de type électrostatiques et interactions VDW. Les paramètres et la complexité d'un CF (le nombre de ressorts et d'interactions pris en compte) déterminent sa précision et sa spécificité.. Les méthodes à base de CF permettent aisément de traiter de l'ordre de $10^{5}$ atomes sur une échelle de temps de $10 \mathrm{~ns}$.

Le présent article développe les idées ci-dessus en les illustrant par des exemples récents. Il est organisé de la façon suivante : le paragraphe 2 traite de l'exemple le plus simple, celui de l'oscillateur harmonique, et fait le lien entre l'énergie et les forces agissant sur les atomes. Le paragraphe 3 introduit la dynamique des réseaux (DR) à partir des équations du mouvement pour un arrangement linéaire de "perles". Le paragraphe 4 présente une revue des diverses méthodes de dynamiques moléculaires (DM). Les approches de DR et de DM sont ensuite comparées dans le paragraphe 5. Le paragraphe 6 présente le calcul de l'énergie totale pour les méthodes de TFD et de CF. Enfin le paragraphe 7 discute des mérites relatifs des $\mathrm{CF}$ et TDF en insistant sur la façon dont ces méthodes peuvent être combinées.

\section{DE L'ÉNERGIE VERS LES FORCES}

Un des exemples les plus simples et pertinents de potentiel énergétique est le potentiel harmonique. Dans ce modèle, la dépendance entre l'énergie $\mathrm{E}$ et le déplacement $\mathrm{x}$ est de type quadratique :

$$
E=\frac{k x^{2}}{2}
$$

L'expression générale de la force $F$ agissant sur la particule qui se trouve dans le puits est :

$$
F=\frac{-d E}{d x}-k x
$$

La constante de rappel $k$ est donc définie par :

$$
k=\frac{-d F}{d x}=\frac{d^{2} E}{d x^{2}}
$$

La particule dans le puits est à l'équilibre pour $x=0$, c'est-à-dire lorsque l'énergie est minimale et lorsque la force de rappel agissant sur la particule est nulle. Si la particule de masse $m$ est déplacée de sa position d'équilibre, elle va osciller perpétuellement à la pulsation $\omega$ :

$$
\omega^{2}=\frac{k}{m}
$$

Ce développement très simple nous permet d'introduire "l'effet de masse" qui permet d'interpréter les vibrations d'un matériau quand un des types d'atomes (voir même l'atome d'un seul site) est remplacé par un autre type d'atome. Si le matériau et son homologue, sont isostructuraux et isoélectroniques (substitution isotopique) alors "l'effet de masse" sur la fréquence est :

$$
\left(\frac{\omega}{\omega^{\prime}}\right)^{2}=\frac{m^{\prime}}{m}
$$

car la surface d'énergie potentielle, caractérisée ici par la constante $k$, est inchangée.

Ce qui rend le potentiel harmonique tellement utile est qu'il est le premier terme non-nul dans le développement limité de la SEP, le terme linéaire étant égal à zéro à la position d'équilibre. En pratique, il se trouve que cette approximation est satisfaisante si la température du cristal est bien inférieure à son point de fusion.

\footnotetext{
${ }^{9}$ En Anglais : FF pour "Force Field".
} 


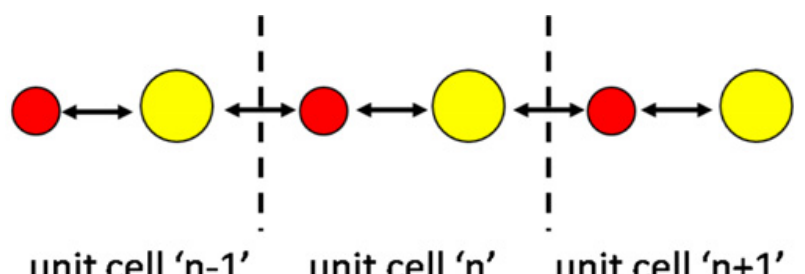

Figure 1. Chaîne diatomique avec les masses $m$ en rouge et $M$ en jaune. La maille périodique est de longueur a.

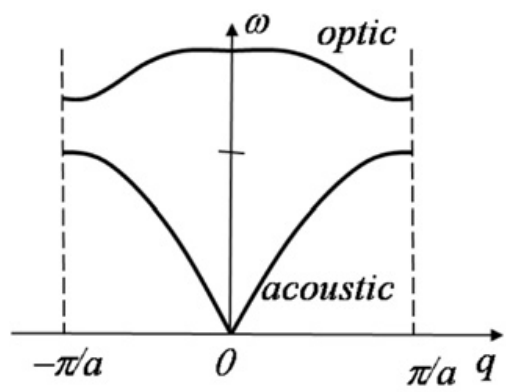

Figure 2. Courbe de dispersion pour une chaîne diatomique.

\section{LA DYNAMIQUE DES RÉSEAUX}

Dans les manuels scolaires, la dynamique des réseaux est souvent introduite à travers une chaîne linéaire d'atomes. Pour une chaîne monoatomique unidimensionnelle, la seule vibration possible est un phonon acoustique (voir ci-dessous). Un second niveau de complexité est de considérer deux atomes de masses différentes $m$ et $M$ placés dans une maille périodique de longueur $a$ avec pour distance interatomique a/2 (cf. figure 1).

On obtient les équations du mouvement des deux atomes de la maille élémentaire $n$ en combinant les expressions précédentes de la force $F$.

$$
\begin{gathered}
F=m a=-k x, \\
m \ddot{x}_{m n}=k\left[x_{M n}+x_{M(n-1)}-2 x_{m n}\right], \\
M \ddot{x}_{M n}=k\left[x_{M n}+x_{M(n+1)}-2 x_{M n}\right] .
\end{gathered}
$$

En faisant l'hypothèse que la solution est une onde plane :

$$
\begin{gathered}
X_{m n}(t)=A_{m} e^{i(q n a-\omega t)}, \\
X_{M n}(t)=A_{M} e^{i(q n a+q a / 2-\omega t)} .
\end{gathered}
$$

on obtient alors un système d'équations en $A_{m}$ et $A_{M}$ qui admet des solutions non nulles si et seulement si son déterminant est égal à zéro :

$$
\left|\begin{array}{cc}
2 k-M \omega^{2} & -2 k \cos \left(\frac{q a}{2}\right) \\
-2 k \cos \left(\frac{q a}{2}\right) & 2 k-m \omega^{2}
\end{array}\right|=0,
$$

avec $q$ le vecteur d'onde correspondant à la longueur d'onde $\lambda=2 \pi / q$.

Ces équations admettent deux solutions pour chaque valeur de $q$. La relation entre la pulsation $\omega$ (souvent dénommée «fréquence » par abus de langage) et le vecteur d'onde $q$ est appelée relation de dispersion. La courbe de dispersion de la chaîne unidimensionnelle diatomique présentée sur la figure 2 
comporte deux branches distinctes. La branche de fréquence la plus basse est la branche acoustique caractérisée par une fréquence $\omega$ nulle à $q=0$. La seconde est la branche optique. Suivant la valeur du vecteur d'onde et la branche de dispersion considérée, différents types de vibrations apparaissent.

Pour les faibles valeurs de $q$, la longueur d'onde des vibrations est très grande devant $a$. Les atomes vibrent en phase pour la branche acoustique et en opposition de phase pour la branche optique. Les ondes sont progressives.

En bord de zone de Brillouin $q=\pi / a$, seul l'atome le plus lourd est en mouvement sur la branche acoustique $\left(\omega^{2}=2 \mathrm{k} / \mathrm{m}\right)$ et seul l'atome le plus léger est en mouvement sur la branche optique $\left(\omega^{2}=2 k / M\right)$. Les ondes sont stationnaires.

En termes de simulations, comme cela sera présenté plus loin, il est important de noter qu'un modèle périodique de longueur de répétition a est capable de décrire des vibrations de longueur d'onde quelconque. En effet, son domaine d'interaction étant $a / 2$, les interactions interatomiques sont limitées à la maille périodique. Dans ce modèle, les vibrations de grande longueur d'onde dépendent seulement des interactions entre plus proches voisins.

A titre purement pédagogique, on peut également définir une supercellule de longueur 2a (quatre particules par maille élémentaire). Le déterminant s'écrit alors :

$$
\left|\begin{array}{cccc}
2 k-M w^{2} & -k e^{i q a / 2} & 0 & -k e^{-k e^{-i q a / 2}} \\
-k e^{-i q a / 2} & 2 k-m w^{2} & -k e^{-i q a / 2} & 0 \\
0 & -k e^{-i q a / 2} & 2 k-M w^{2} & -k e^{-i q a / 2} \\
-k e^{i q a / 2} & 0 & -k e^{i q a / 2} & 2 k-m w^{2}
\end{array}\right|=0 .
$$

Il est également possible de prendre en compte les interactions entre seconds voisins. Ce degrès supplementaire de complexité a pour effet de supprimer les eléments nuls du déterminant.

$\mathrm{Si}$ on généralise cette approche au cas tridimensionnel, les éléments de la MD prennent alors la forme :

$$
M D_{i j}=\frac{k_{i j}}{\sqrt{m_{i} m_{j}}} e^{i\left[\boldsymbol{q}\left(\boldsymbol{r}_{i}-\boldsymbol{r}_{j}\right)\right]} .
$$

avec les indices $\{i, j\}$ associés aux $N$ atomes de la maille élémentaire de masse $m_{j}$ et de coordonnées cartésiennes $\boldsymbol{r}_{j}=\{x, y, z\}$. La matrice $k_{i j}$ est appelée "matrice hessienne" du système.

La diagonalisation de la MD donne $3 \mathrm{~N}$ branches qui sont composées de 3 branches acoustiques et de $3 \mathrm{~N}-3$ branches optiques. Les vecteurs propres correspondants décrivent les déplacements relatifs des atomes/particules, et le déplacement physique est obtenu en pondérant ce résultat par la masse, c'est-à-dire en divisant chaque composante du vecteur propre par la racine carrée de la masse atomique correspondante.

\section{Méthode directe pour le calcul des phonons}

Une méthode générale, et conceptuellement simple, de calcul des phonons est la méthode directe - ou approche par supercellules - qui conduit à une détermination complète de la MD. Cette approche est implémentée dans le logiciel PHONON [1] couramment utilisé à l'ILL, et dans le logiciel apparenté NMScatt [2] développé pour les systèmes biomoléculaires comme l' ADN [3]. Les étapes de ces méthodes directes sont :

- déterminer la structure à l'équilibre du système périodique (maille du cristal);

- construire la supercellule qui inclut toutes les interactions interatomiques (idéalement un cube d'au moins $\sim 10 \AA$ de côté);

- générer l'ensemble des structures pour lesquelles chaque atome non cristallographiquement équivalent est déplacé le long de directions cartésiennes non équivalentes (typiquement $3 \mathrm{~N}$ déplacements si $\mathrm{N}$ est le nombre d'atomes non-équivalents). 
- calculer les forces interatomiques pour chaque structure;

- construire la MD à partir de ces forces, des amplitudes et des symétries des déplacements atomiques;

- diagonaliser la MD pour chaque valeur de $q$.

Les fréquences de vibration obtenues de cette façon doivent être supérieures ou égales à zéro. Les fréquences négatives éventuelles sont en fait des fréquences imaginaires puisque les valeurs propres de la MD sont en $\omega^{2}$, et sont l'indication des problèmes numériques ou physiques suivants :

- la structure à l'équilibre est métastable et l'un des déplacements atomiques conduit le système vers une autre structure stable;

- la structure à l'équilibre n'est pas suffisament bien optimisée et les forces résiduelles sont non nulles;

- les forces de rappel sur les atomes déplacés doivent être de l'ordre de 0,5 eV/Å pour un déplacement de $0,05 \AA$;

- la supercellule ne contient pas toutes les interactions interatomiques, ce qui peut être le cas lorsque les forces électrostatiques sont importantes.

Les fréquences des vibrations données par la MD peuvent être utilisées pour calculer les courbes de dispersion et la densité d'état totale des vibrations, mais aussi pour faire le lien avec des quantités thermodynamiques comme la capacité calorifique, l'entropie, l'énergie libre, etc. A partir des vecteurs de déplacement on obtient des densités d'états vibrationnels partielles $(\mathrm{DEVp})^{10}$ - par exemple par type d'atomes - permettant ensuite de calculer la densité d'états vibrationnels généralisée (DEVG) ${ }^{11}$ :

$$
\begin{gathered}
D E V G=\Sigma_{i} \frac{\sigma_{i}}{m_{i}} D E V p_{i}, \\
D E V p_{i}=\Sigma_{j} e_{i j}^{2},
\end{gathered}
$$

avec $e_{i j}$ les composantes des vecteurs propres des atomes de type $i$. Les vecteurs propres sont également utilisés pour déterminer l'intensité spectrale, que ce soit pour la diffusion cohérente le long des courbes de dispersion (cf figure 3) ou pour la diffusion incohérente d'échantillons orientés [4] ou de poudres [5].

\section{Théorie de la réponse linéaire adaptée au calcul des phonons}

Une méthode très répandue de calcul $a b$ initio de dynamique des réseaux est basée sur la théorie de réponse linéraire (RL) ${ }^{12}$ ou sur la théorie de pertubation de la densité fonctionnelle (TPDF) ${ }^{13}$ [7] (cf. tableau 1). Sa pertinence va au-delà de l'étude des propriétés vibrationnelles car la RL permet le calcul analytique de la dérivée seconde de l'énergie totale par rapport à une perturbation donnée. Ainsi la pertubation de sites ioniques donne la matrice dynamique et les phonons. Bien que construire la RL ne nécessite pas une supercellule, un calcul de TPDF est souvent plus gourmand en temps de calcul que son équivalent par la méthode directe. Toutefois l'avantage de la méthode RL est de prendre en compte les interactions électrostatiques à grande distance qui s'étendent au-delà d'une supercellule classique. En contre-partie la RL impose de façon catégorique l'approximation harmonique, alors que les déplacements atomiques utilisés par la méthode directe peuvent être adaptés de façon à explorer le cas anharmonique.

\footnotetext{
${ }^{10}$ En Anglais : pVDOS pour "partial Vibrational Density Of States".

11 En Anglais : GVDOS pour "Generalised Vibrational Density Of States".

12 En Anglais : LR pour "Linear Response".

13 En Anglais : DFPT pour "Density Functional Perturbation Theory".
} 


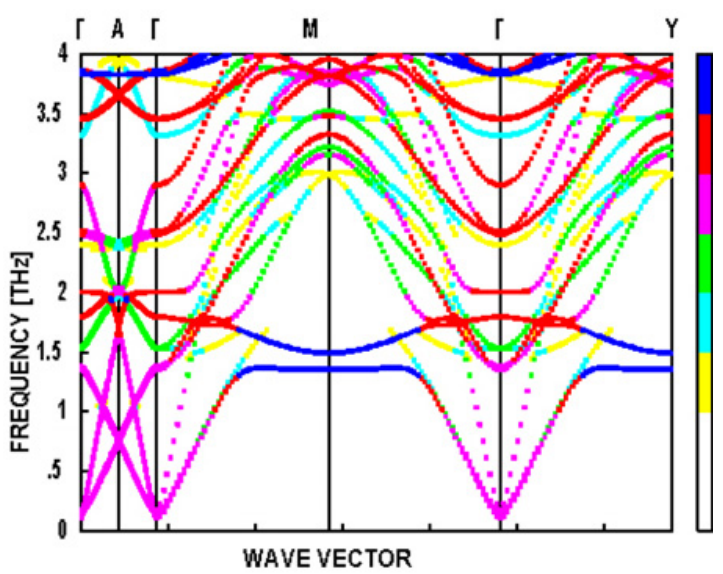

Figure 3. Courbes de dispersion pour les directions de haute symétrie de la structure de type pyrochlore de l'oxyde de strontium-gallium $\left(\mathrm{SrGa}_{\mathrm{x}} \mathrm{O}_{\mathrm{y}}\right)$. Le code de couleur indique l'intensité des modes. On voit par exemple que, à $\sim 6 \mathrm{meV}(1.5 \mathrm{THz})$, l'intensité spectrale sera dominée par les modes aux points $\mathrm{M}$ et $\mathrm{Y}$ [6].

\section{Dynamique des réseaux moyennée pour une poudre}

L'approche précédente s'applique aux monocristaux alors que la plupart des échantillons étudiés pas DIN sont sous forme de poudres. Afin de palier à ce problème, nous avons récemment formalisé une dynamique des réseaux moyennée pour les poudres (DRMP) ${ }^{14}$. Quand on extrait la DEVG à partir des données de DIN, on fait cela dans l'approximation incohérente qui suppose que l'intensité inélastique croît de façon monotone en $Q^{2}$. En fait, dans le cas d'échantillons dont les atomes sont surtout des diffuseurs cohérents, $S(Q, \omega)$ ne croît pas de façon monotone avec $Q^{2}$ et la variation en intensité contient des informations sur la dispersion des modes de vibration ainsi que sur les couplages interatomiques. Dans les calculs usuels sur des monocristaux, on étudie la variation en intensité le long des courbes de dispersion des directions de haute symétrie (voir plus haut). Pour la DRMP au contraire, on ne s'intéresse pas à une direction particulière. Le facteur de structure cohérent est déterminé à partir d'un ensemble complet de points distribués au hasard dans la zone de Brillouin et couvrant le domaine en Q qui nous intéresse [8].

\section{DYNAMIQUE MOLÉCULAIRE (DM)}

La DM décrit le mouvement perpétuel des atomes d'un système en fonction du temps à des conditions de température et de pression données. Une trajectoire de DM est un ensemble de structures dans laquelle chaque atome a une position $\boldsymbol{r}$ et une vitesse $\boldsymbol{v}$. Chaque nouvelle structure est calculée à partir des forces instantanées $\boldsymbol{F}$ agissant sur les atomes de la structure précédente. L'accélération $\boldsymbol{a}$ sur chacun des atomes peut s'écrire :

$$
\boldsymbol{a}=\frac{\boldsymbol{F}}{m} .
$$

Une simple intégration des équations du mouvement donne les positions des atomes de la nouvelle structure :

$$
\boldsymbol{r}(t+\mathrm{d} t)=\boldsymbol{r}(t)+\boldsymbol{v}(t) \mathrm{d} t+\frac{\boldsymbol{a}(t)}{2 \mathrm{~d} t^{2}}
$$

\footnotetext{
14 En Anglais : PALD pour "Powder Averaged Lattice Dynamics".
} 

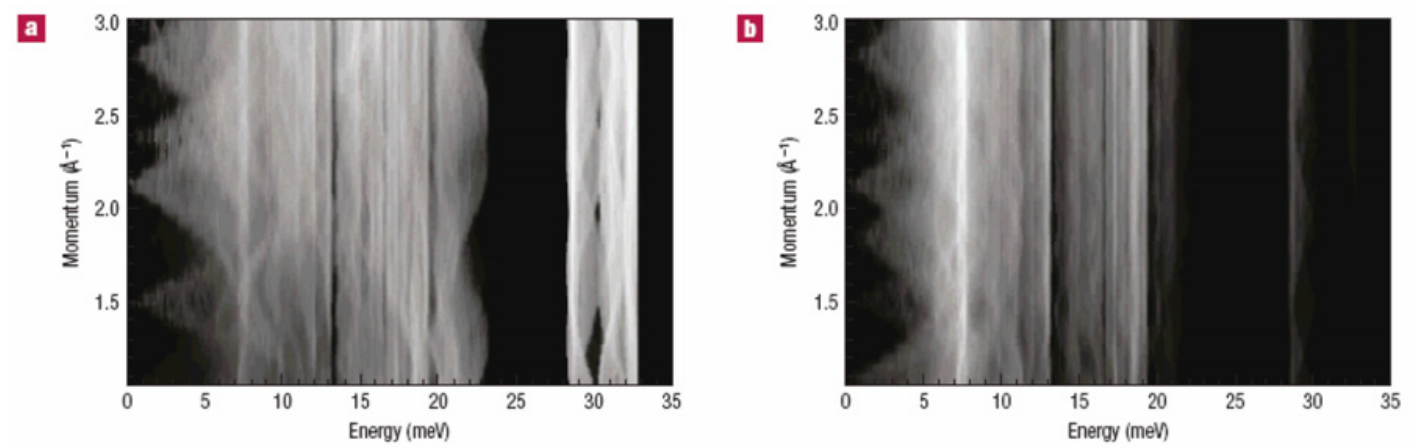

Figure 4. Cartes de $S(Q, \omega)$ en échelle logarithmique pour une skuttérudite dont les atomes hôtes La et Ce sont "emprisonneés" dans un réseau Fe-Sb [9]. (a) montre la réponse totale, tandis que (b) montre la réponse des seuls atomes de La. Ici, c'est la diffusion cohérente des trois types d'atomes qui domine. On note une forte dépendance en $Q$ de l'intensité (une diffusion incohérente majoritaire donnerait une dépendance monotone en $Q^{2}$ ). On voit immédiatement les phonons acoustiques à $\omega \approx 0$, notamment ceux issus des pics de Bragg à $1,5 \AA^{-1}$ et $2,2 \AA^{-1}$.

ainsi que leurs vitesses :

$$
v(t+d t)=v(t)+a(t) d t,
$$

avec $\boldsymbol{r}, \boldsymbol{v}$ et $\boldsymbol{a}$ les vecteurs décrivant respectivement les positions, vitesses et accélérations.

Le pas de la simulation $d t$ doit être significativement plus court (environ 5 fois) que la période des fréquences de vibration les plus élevées. Par exemple, les fréquences de vibration les plus élévées des systèmes contenant de l'hydrogène $\mathrm{H}$ étant de $\sim 400 \mathrm{meV}, d t$ est typiquement de l'ordre de 1 fs. Pour des systèmes composés d'atomes plus lourds, ou pour des systèmes moléculaires considérés comme rigides ou constitués d'unités partiellement rigides, $d t$ peut atteindre 5-10 fs.

Le temps total de simulation est égal à $d t$ multiplié par le nombre de pas de simulation. Le choix de $d t$ doit se faire avec précaution : $d t$ étant le temps durant lequel les atomes suivent une trajectoire balistique, il ne doit pas être trop grand sans quoi les distances interatomiques peuvent devenir très courtes, les forces et les accélérations très grandes, ce qui rend alors la simulation instable.

Les simulations de DM ont pour structure de départ la structure à l'équilibre. Dans cette structure particulière, les distances interatomiques ont été préalablement optimisées de façon à ce que la résultante des forces sur chaque atome soit proches de zéro. Ce système statique est alors mis en mouvement par une impulsion initiale : on attribue aux atomes une distribution aléatoire de vitesses correspondant à l'énergie cinétique de la température voulue.

On appelle $N V E, N V T, N P T$ ou $N P H$ des simulations de DM conservant diverses combinaisons de grandeurs thermodynamiques comme le volume $(V)$, l'énergie $(E)$, l'enthalpie $(H)$, la température $(T)$, ou la pression $(P) . N$ est le nombre d'atomes dans la boîte de simulation, lequel reste toujours constant.

Pour des conditions expérimentales usuelles (pression et température fixées), les simulations NPT sont les plus réalistes. Pour que $P$ et $T$ soient contrôlées, le système est connecté à un thermostat et un baromètre qui interagissent avec la dynamique atomique, généralement avec fréquence fixée par l'utilisateur. Toutefois le mode de simulation le plus simple et le plus "sûr" est l'ensemble canonique $N V E$ car il n'y a pas de couplage avec un bain externe.

Une fois le système mis en mouvement par l'impulsion initiale, les premières structures simulées passent par une phase de mise en équilibre au terme de laquelle les grandeurs caractéristiques ( $\mathrm{V}, \mathrm{P}$, $\mathrm{T}, \ldots$...) non-conservées doivent se stabiliser, en oscillant autour de leurs valeurs moyennes. Bien que la structure initiale soit considérée comme étant à l'équilibre, l'impulsion est rarement conforme à la dynamique réelle et la phase de mise à l'équilibre peut alors être considérée comme permettant une redistribution de l'énergie entre les modes normaux du système. La phase de mise à l'équilibre et la 


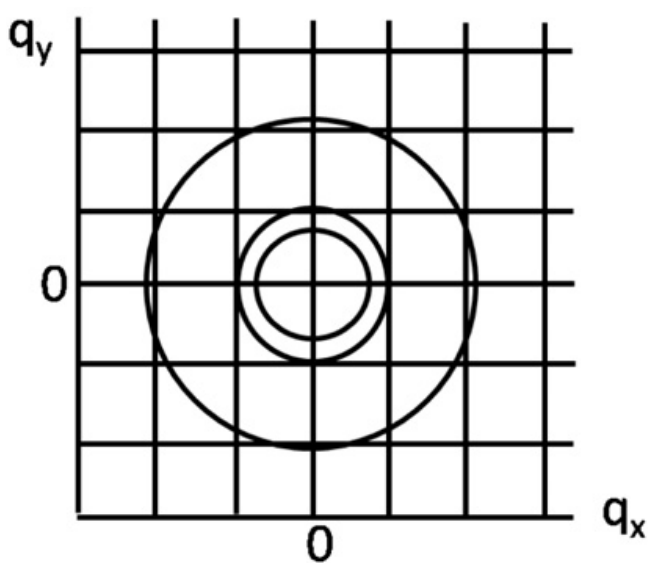

Figure 5. Pavage bidimensionnel dans l'espace des vecteurs d'onde (pas $\delta Q=2 \pi / L$ avec $\mathrm{L}$ dimension de la boîte de simulation). Le plus petit anneau représenté $Q=\sqrt{q_{x}^{2}+q_{y}^{2}}<2 \pi / L$ n'intercepte aucun point du réseau réciproque. Le second intercepte quatre points, tandis que le plus grand passe à proximité de huit points. En pratique, l'information suivante est requise lorsqu'on échantillonne l'espace des Q : largeur en Q et nombre de points ( $\left.n_{Q \text { Sample }}\right)$ à échantillonner dans chaque domaine en $Q$. Pour les petits $Q$, le nombre de points du réseau réciproque dans un domaine de $\mathrm{Q}$ peut être inférieur à $n_{Q \text { Sample }}$, auquel cas tous les points disponibles sont échantillonnés.

phase de production qui s'en suit doivent être traitées par deux simulations distinctes. Dans le cas d'une simulation unique, il faut absolument vérifier le début, le milieu et la fin et s'assurer de la stabilisation des valeurs macroscopiques et microscopiques.

Les observables de DIN sont extraites des trajectoires via les fonctions de corrélation dépendantes du temps. La DEV [voir plus haut pour la définition de la DEV et de la DEVG] est donnée par la transformée de Fourier de la fonction d'autocorrélation des vitesses $V(t)$ :

$$
V(t)=\left\langle\boldsymbol{v}_{i}\left(t_{0}\right) \cdot \boldsymbol{v}_{i}\left(t_{0}+t\right)\right\rangle .
$$

On peut ensuite calculer les DEVp par atome et pour chaque direction cartésienne, ce qui revient à extraire les modes normaux sans connaître la relation de phase entre les déplacements atomiques. Dans ce cas, à la manière de l'approche de DR, la DEVG peut alors être calculée comme la somme pondérée des DEVp.

$$
D E V G=\Sigma_{i} \sigma_{i} p D E V p_{i}
$$

Contrairement à l'équation (15), les masses atomiques n'apparaissent pas dans cette expression car elles sont partie intégrante de la simulation de DM et sont donc incluses dans la DEVp. $S(Q, \omega)$ est calculé à partir des trajectoires $\boldsymbol{r}_{i}(t)$ via les fonctions intermédiaires de diffusion :

$$
\begin{gathered}
I_{c o h}(\boldsymbol{Q}, t)=\frac{1}{N} \sum_{i} \sum_{j \neq i}\left\langle\mathrm{e}^{i \boldsymbol{Q} \cdot \boldsymbol{r} i(0)} \mathrm{e}^{-i \boldsymbol{Q} \cdot \boldsymbol{r} j(t)}\right\rangle . \\
I_{i n c}(\boldsymbol{Q}, t)=\frac{1}{N} \sum_{i}\left\langle\mathrm{e}^{i \boldsymbol{Q} \cdot \boldsymbol{r i}(0)} \mathrm{e}^{-i \boldsymbol{Q} \cdot \boldsymbol{r} i(t)}\right\rangle .
\end{gathered}
$$

$Q$ peut être choisi selon une direction donnée afin de tester une dynamique spécifique, comme, par exemple, les phonons longitudinaux de l'ADN en alignant $\mathrm{Q}$ selon les axes de l'hélice. $Q$ peut aussi être pris comme un ensemble aléatoire de directions et la réponse est alors une moyennisation de type poudre.

Pour une boîte de simulation de type poudre et de côté $L$, la valeur minimale de $Q$ accessible est $2 \pi / L$ (cf. figure 5). Si la maille de simulation est la maille cristallographique de coté $a$, alors seuls 


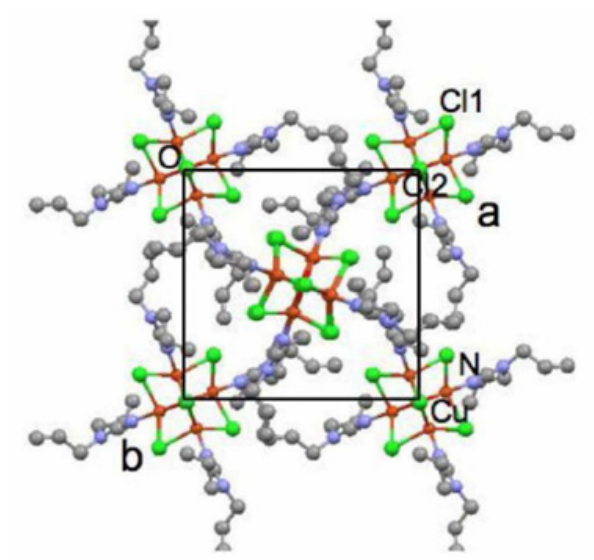

Figure 6. Projection a-b de la structure de $\mathrm{Cu}_{4} \mathrm{OCl}_{6} \mathrm{daca}_{4}$.

les phonons de longueur d'onde inférieure et commensurable à $a$ sont échantillonnés. Toutefois, si on construit une super-maille, on obtiendra des phonons de plus grande longueur d'onde, commensurables avec cette super-maille. Cette approche est donc à opposer à la DR qui permet de calculer de façon précise les phonons de toutes longueurs d'ondes, à condition que la super-maille contienne toutes les interactions interatomiques.

A l'ILL, nous utilisons le code nMoldyn [10] pour analyser les trajectories de DM et nous participons à son développement.

\section{Exemple :}

Les excitations magnétiques du cuivre tétraèdrique dans $\mathrm{Cu}_{4} \mathrm{OCl}_{6} \mathrm{daca}_{4}$ (cf. figure 6) ont été étudiées par DIN [11]. Des calculs de DR ont alors été tentés afin de faciliter la séparation des signaux magnétiques et des phonons. Malheureusement, ils ont systématiquement conduit à des modes imaginaires du fait de la complexité de la structure électronique associée aux électrons de coeur des atomes d'oxygène entourés par un tétraèdre de cuivre et un octaèdre de chlore, mais aussi à cause de la souplesse des ligands délocalisés et couplés. Des simulations de DM à base de TDF ont ensuite été effectuées à partir de la maille cristallographique. Elle ont permis de montrer que la fréquence la plus basse des modes optiques était de $2 \mathrm{meV}$. Les excitations observées en dessous de cette fréquence sont vraisemblablement purement magnétiques. La figure 7 montre la DEVp déduite d'une simulation de DM de 27 picosecondes.

\section{COMPARAISON ENTRE LES MÉTHODES DR ET DM}

Les points suivants présentent les avantages respectifs des méthodes DR et DM lors du calcul de la dynamique d'un système d'atomes:

- Structure: la méthode DR est basée sur une seule structure, celle à l'équilibre, tandis que le point de départ de la méthode DM est une structure approximant la structure à l'équilibre. Par contre, la méthode DM peut explorer un ensemble de structures correspondant à des minima locaux de la SEP.

- Température: dans la méthode $\mathrm{DR}$, la température est à $0 \mathrm{~K}$ puisque les atomes sont au minimum de leur énergie potentielle. Néanmoins, les variations du réseau cristallin sous l'effet de la température peuvent être mesurées en procédant à plusieurs calculs de phonons paramétrés par les paramètres 

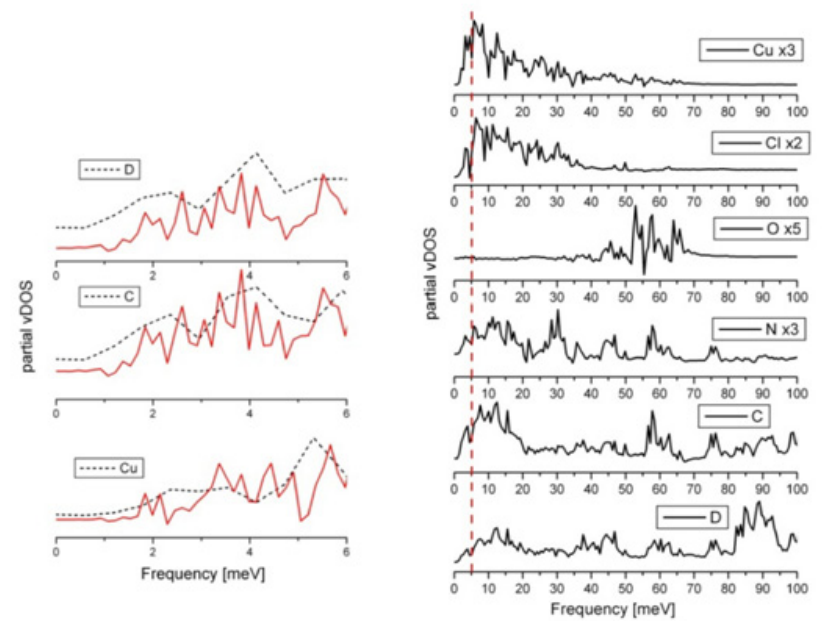

Figure 7. DEVp pour les différents types d'atomes dans $\mathrm{Cu}_{4} \mathrm{OCl}_{6}$ daca $_{4}$ : pour un domaine de fréquence étendu (courbes de droite), et à basse fréquence (courbes de gauche). Le panneau de gauche montre les résultats de simulation de $\mathrm{DM}$ au bout de $10 \mathrm{ps}$ (courbes pointillées noires) et à une meilleure résolution (courbes continues rouges) correspondant à une simulation complète de 27 ps. La qualité statistique des résultats de DM dépend fortement de la longueur de la simulation.

de réseau correspondants. Cette approche est appelée approximation quasi-harmonique $(\mathrm{AQH})^{15}$. Dans la méthode DM, la température est directement déterminée à partir de l'énergie cinétique du système.

- Approximation harmonique: cette approximation est obligatoire dans la méthode DR tandis que des contributions non harmoniques à la SEP sont accessibles avec la méthode DM.

- Modes normaux: dans la méthode DR, ils peuvent être obtenus pour n'importe quel vecteur d'onde. Ceci permet d'obtenir une courbe de dispersion complète. Dans la méthode DM, l'échantillonnage de la zone de Brillouin est limité aux multiples de la plus petite valeurs de Q correspondant aux dimensions de la boîte de simulation. En principe, les modes normaux ne sont pas accessibles à la méthode DM. Toutefois, ils peuvent être obtenus à l'aide d'approches basées sur l'analyse en composantes principales [12]. Si tel n'est pas le cas, la DEV partiel représente le niveau d'information le plus détaillé.

- Modes imaginaires: dans la méthode DR, ils peuvent être d'origine physique ou numérique. Par contre, les fonctions de corrélation utilisées pour l'analyse des trajectoires issues de la méthode DM ne peuvent pas engendrer de fréquences négatives.

\section{Exemple :}

Dans le cas du 3,5-pyridine acide dicarboxylique [13] (cf Figure 8) les méthodes DR et DM ont toutes deux été utilisées pour étudier l'influence des vibrations du réseau cristallin dans la migration du proton le long de la liaison hydrogène N-H. . . à température croissante.

La méthode DR a été utilisée pour déterminer les vibrations moléculaires du 3,5-PADC à basse température (Figure 9). Etant basée sur l'approximation harmonique, la méthode DR ne permet pas d'obtenir d'informations sur les parties anharmoniques et dépendantes de la température de la SEP. A cet effet, des simulations de DM couplée à la DFT ont été réalisées à des températures allant de

15 En Anglais: QHA pour “Quasi-Harmonic Approximation”. 


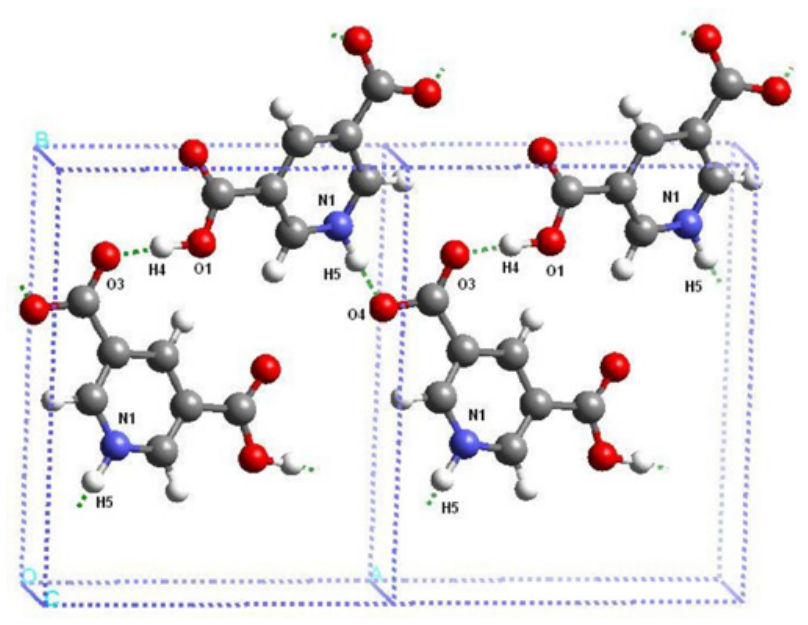

Figure 8. Structure cristalline de la molécule 3,5-PADC montrant la liaison hydrogène N-H..O.
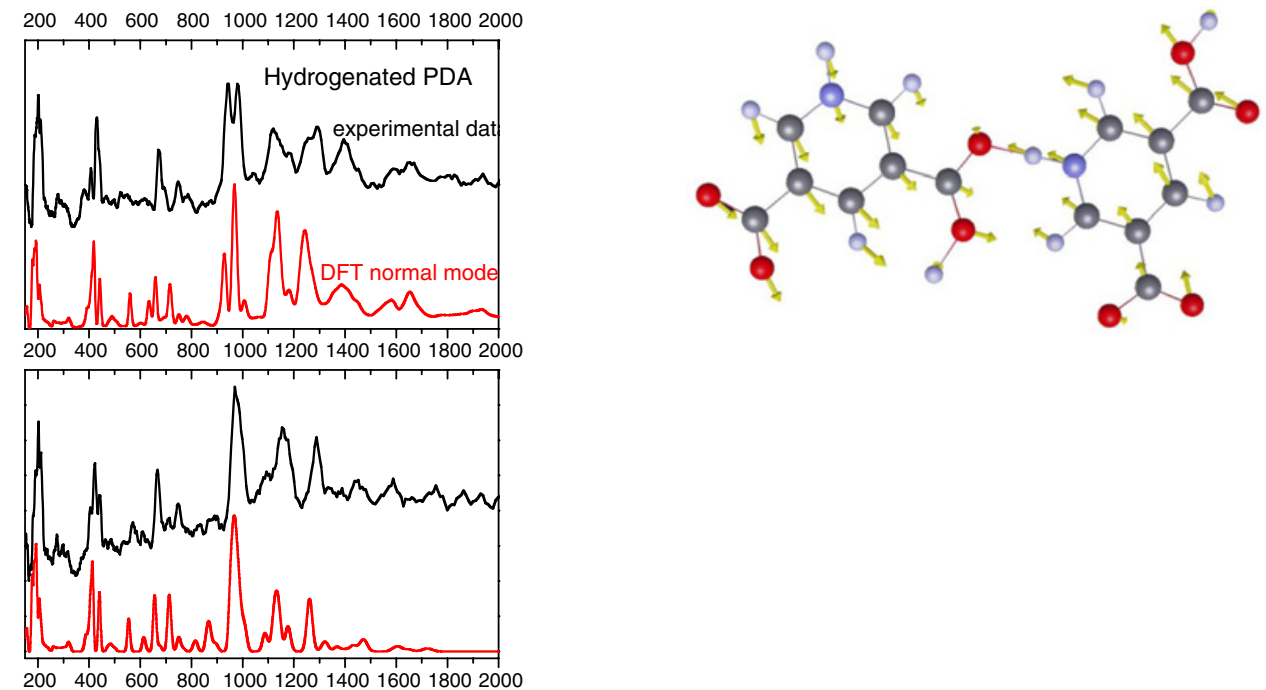

Figure 9. Comparaison entre le spectre de diffusion inélastique expérimental et calculé avec la méthode DR pour le 3,5-PADC, protoné (en haut à gauche) et pour le 3,5-PADC avec liaison hydrogène N-H..O deutérée (en bas à gauche). Le même hessien (matrice des constantes de forces) a été utilisé pour les deux systèmes. La seule différence concernait les masses atomiques. Un mode à basse fréquence permettant le transfert de proton est présenté à droite.

$50 \mathrm{~K}$ à $300 \mathrm{~K}$. Elles ont montré la migration du proton mesurée expérimentalement à $300 \mathrm{~K}$ (Figure 10). La transformée de Fourier de l'évolution temporelle de la longueur de la liaison N-H a notamment permis de mettre en évidence deux modes à 120 et $150 \mathrm{~cm}^{-1}$ responsables de la migration du proton. Les caractéristiques de ces modes ont été obtenues à partir des simulations de DR (cf Figure 9). La fréquence de ces modes leur permet d'être activés à température ambiante (température à laquelle a été observé le transfert de proton sous sa forme complète) et de moduler la géométrie de la liaison hydrogène courte. 


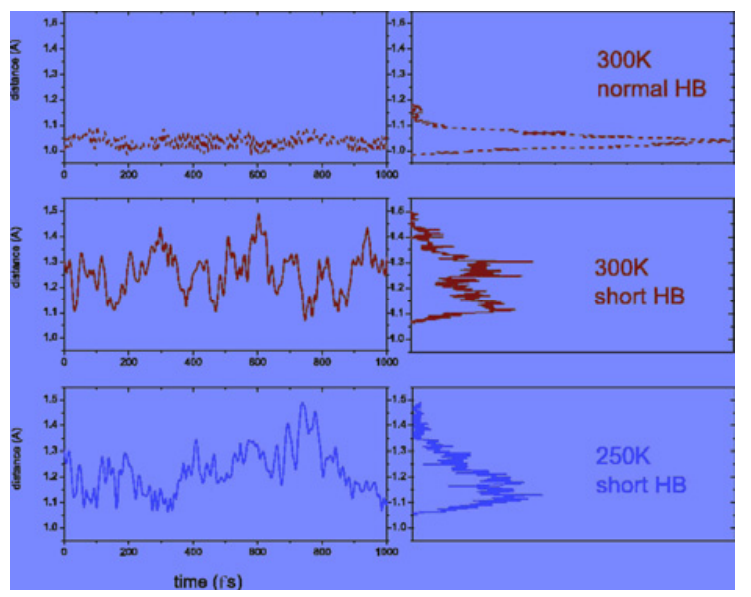

Figure 10. Evolutions temporelles et histogrammes de la longueur de la liaison N-H (4 figures du bas). A $250 \mathrm{~K}$, le début de la migration du proton est clairement caractérisé par la présence d'une asymétrie de l'histogramme vers les grandes longueurs. A $300 \mathrm{~K}$, l'histogramme montre deux pics, caractéristiques de deux positions (méta-)stables pour le proton. L'évolution temporelle de la longueur de la liaison $\mathrm{N}-\mathrm{H}$ montre des oscillations quasi-périodiques dont les fréquences ont pu être obtenues par transformée de Fourier. A titre de comparaison, les données pour la liaison hydrogène $\mathrm{O}-\mathrm{H}$. . O sont montrées dans les deux figures du haut.

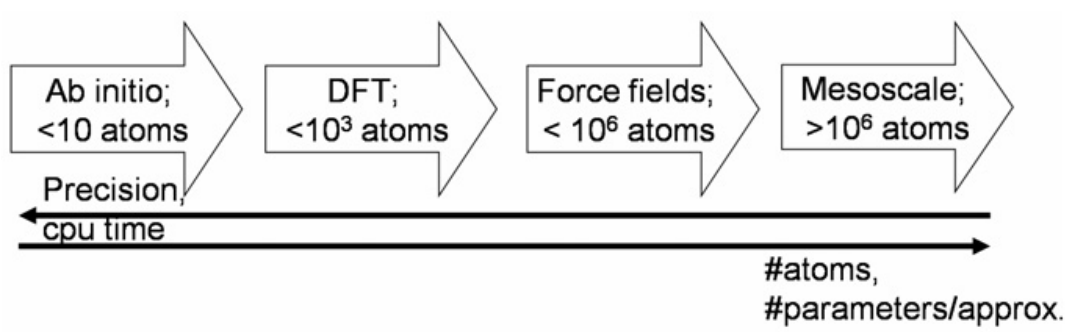

Figure 11. Méthodes disponibles pour les calculs d'énergie totale.

\section{CALCULS DE L'ÉNERGIE TOTALE}

Le but de ce paragraphe est de présenter les grandes méthodes utilisées pour le calcul de l'énergie totale d'un système d'atomes en interaction qui, comme nous l'avons vu précédemment, est la clef de voute des méthodes DR et DM. La figure 11 donne un aperçu des méthodes de simulations disponibles, des méthodes $a b$ initio, les plus précises, aux méthodes mésoscopiques particulièrement adaptées aux systèmes de taille importante. Dans les méthodes $a b$ initio et TFD, la structure électronique du système étudié est déterminée. Dans les méthodes de type CF ou mésoscopiques, les interactions atomiques sont calculées à partir de fonctions relativement simples dont les paramètres représentent implicitement les effets électroniques du système. Nous mettrons ici l'accent sur les méthodes TFD et $\mathrm{CF}$ dans la mesure où celles-ci permettent la simulation d'une grande variété de systèmes pour lesquels des mesures expérimentales en DIN sont disponibles. Il existe un grand nombre de programmes utilisant les méthodes TFD et CF. Nous ne considérerons pas ici les méthodes dites ab initio complètes car, en pratique, le gain de précision qu'elles apportent par rapport à la TFD se fait au détriment d'une réduction drastique de la taille du système simulable. A l'opposé, les méthodes mésoscopiques présentent l'avantage de permettre la simulation de la dynamique basse-fréquence de systèmes de taille très importante comme les systèmes biomoléculaires mais la paramétrisation de ces méthodes est une 
tâche complexe car celle-ci doit décrire l'interaction non plus entre des atomes mais entre des ensembles d'atomes.

\section{Théorie de la Fonctionnelle de Densité (TFD)}

Le théorème de Hohenberg et Kohn (a) donne un cadre théorique pour la réduction du problème à $\mathrm{N}$-corps de $\mathrm{N}$ électrons avec $3 \mathrm{~N}$ coordonnées spatiales aux 3 coordonnées spatiales permettant la description de la densité mono-électronique et (b) définit la fonctionnelle d'énergie avec comme résultat principal la preuve que la densité électronique dans l'état fondamental minimise l'énergie décrite par cette fonctionnelle d'énergie. Kohn et Sham ont formulé par la suite la description de l'interaction entre ions et électrons en terme d'électrons indépendants se déplaçant sous l'action d'un potentiel externe du aux ions. Ceci est exprimé par l'équation suivante (KS-TFD).

$$
E_{k s}[\rho(r)]=T[\rho(r)]+E_{e s}[\rho(r)]+E_{e x t}[\rho(r)]+E_{x c}[\rho(r)],
$$

où $\rho(r)$ est la densité mono-électronique dépendante de la position. Si la fonction d'onde électronique est connue alors $\rho(r)=\psi \psi^{\prime}$. Les quatre membres de droite de cette équation sont les suivants: $T$ décrit l'énergie cinétique; $E_{e s}$ décrit les interactions électrostatiques entre les ions; $E_{\text {ext }}$ décrit les interactions électrostatiques entre les ions et les électrons et enfin, $E_{x c}$ représente l'énergie d'échange et de corrélation des électrons.

Dans la méthode TFD, les contributions d'échange et de corrélation à l'énergie totale sont estimées à partir de fonctionnelles de densité de paramétrisation et de formulation mathématique différentes. Dans la théorie de Hartree-Fock originelle, où le terme de corrélation n'est pas pris en compte, la contribution d'échange est la conséquence de la nature du déterminant de Slater polyélectronique. Par la suite, des méthodes basées sur la méthode d'interaction de configuration ont permis d'introduire des corrélations statiques et dynamiques. Mais, à la différence de la TFD, ces méthodes sont encore très limitées par la taille du système. Les fonctionnelles d'échange et de corrélation donnant $E_{x c}$, peuvent être déterminées à partir de deux stratégies (voire philosophies) différentes: empirique/semiempirique, à partir de modèles de régression spectrochimiques (BLYP, BP), ou à partir de raisonnements purement mathématiques (PBE). Il existe une grande variété de fonctionnelles disponibles : locales, semi-locales et non-locales. Le but ultime étant d'avoir une fonctionnelle non-locale afin d'obtenir la plus grande précision possible. Dans ce contexte, plusieurs fonctionnelles locales ont été construites: les fonctionnelles locales dans lesquelles la dépendance repose uniquement sur $\rho(\mathrm{r})$ (LDA); les fonctionnelles semi-locales dépendantes de $\rho(\mathrm{r})$ et de son gradient (GGA: BLYP, PW, PBE,...); les fonctionnelles hybrides qui sont de type GGA avec en plus une certaine contribution de l'énergie d'échange exacte d'Hartree-Fock (B3LYP, PBE0,...) et les fonctionnelles meta GGA dépendantes de $\rho(\mathrm{r})$ de son gradient et de son énergie cinétique (TPSS, mPWKCIS, ...).

D'un point de vue pratique les équations de KS sont résolues de manière itérative auto-consistante. A partir d'une hypothèse initiale sur la valeur de la densité électronique, les termes de l'équation (KS-TFD) sont déterminés et l'équation peut alors être résolue pour donner une nouvelle valeur de la fonctionnelle $\rho$. Ce processus est répété jusqu'à qu'un critère de convergence soit satisfait. Ce critère étant que la variation d'énergie totale du système doit être inférieure à une valeur $\mathrm{dE}$, typiquement de l'ordre de $10^{-6} \mathrm{eV}$. Les forces agissant sur les atomes sont alors définies par les dérivées de l'énergie totale par rapport aux positions atomiques correspondantes. Ces forces sont appelées forces d'HellmannFeynman. Lors d'une optimisation de structure, ce sont les forces HF qui sont minimisées à zéro (avec une tolérance numérique généralement de l'ordre de $10^{-4} \mathrm{eV} / \AA$ ). Ces forces sont également à la base des simulations de DM pour l'évolution de la structure.

Les derniers développements dans la méthode TFD concernent la fonctionnelle XC et ce qu'on appelle la réduction linéaire. L'approximation (semi-) locale de la densité pour $E_{x c}$ (LDA or GGA) peut donner des résultats erronés au niveau de la description de la corrélation des orbitales électroniques localisées $d$ et $f$, qui se manifestent par une sous-estimation de l'énergie de la bande interdite ainsi 
que par une surestimation de la covalence. Ce problème peut être corrigé dans l'esprit du modèle de Hubbard avec l'introduction d'un potentiel coulombien in situ $U$ et d'un paramètre d'échange $J$, où $U-J$ est un paramètre empirique effectif. Cette approche est appelée LDA + U. Une approche alternative mais beaucoup plus coûteuse impliquent l'utilisation de fonctionnelles hybrides. De manière générale, lors de l'évaluation de la précision d'un calcul de TFD sur la dynamique d'un système d'atomes (ce qui, rappelons-le, est l'objet de cet article), la structure électronique sous-jacente devrait toujours être vérifiée.

Théoriquement, la méthode TFD calculant une densité mono-électronique, le temps de calcul devrait varier de façon linéaire avec le nombre d'atomes du système. Par exemple, si le nombre d'atomes double le temps de calcul devrait être approximativement deux fois plus long. Hélas, en pratique, cette dépendance n'est pas en $N$ mais plutôt en $N^{2}$ voire $N^{3}$. Ceci est lié à la décomposition de la densité électronique sur une base orthonormée de fonctions d'onde mono-électroniques et à la diagonalisation de matrices afin de résoudre l'équation de Schroedinger et d'obtenir la fonction d'onde (et sa densité associée) de l'état fondamental ainsi que son énergie. Toutefois, la linéarisation est possible sans perte de précision, en reformulant la TFD en terme de matrice de densité "near-sighted" [14]. Le coût de cette reformulation devient avantageux pour les systèmes comportant plus de 500 atomes. Pour un nombre inférieur, l'implémentation traditionnelle de la TFD est plus intéressante. Plusieurs programmes proposant la linéarisation sont disponibles à ce jour parmi lesquels SIESTA [15] et ONETEP [16]. Ils permettent la simulation de systèmes allant jusqu'à $10^{5}$ atomes sur des machines parallèles.

\section{Considérations pratiques :}

En plus des considérations théoriques précédemment évoquées, un certain nombre de termes et de choix doivent être considérés lors d'un calcul de structure électronique.

- Méthode: Hartree-Fock ou TFD.

- Base: il s'agit des fonctions utilisées pour la construction de la fonction d'onde électronique ou de la densité électronique. En général, ces fonctions sont des orbitales atomiques localisées, telles que des gaussiennes. Dans les programmes de TFD utilisés en physique du solide, des bases constituées d'ondes planes peuvent être utilisées ce qui a pour avantage de permettre la détermination de la densité électronique en TOUT point du système périodique. La taille du jeu de base est l'un des facteurs critiques intervenant dans la précision du calcul.

- Points-k: les calculs dans l'état solide sont effectués dans les espaces réels et réciproques, ce dernier étant défini par un maillage de points dans l'espace des vecteurs d'onde. Une valeur initiale de $0.1 \AA^{-1}$ comme espacement entre deux points de ce maillage est considérée comme raisonnable. La convergence dépend également de cette valeur d'espacement. Par conséquent, le nombre de points $\mathrm{k}$ doit parfois être augmenté en gardant à l'esprit que le temps de calcul croit linéairement avec ce nombre de points.

- Pseudopotentiels: un calcul de TFD peut être effectué en considérant tous les électrons d'un atome. Cependant, les électrons de cœur n'interviennent pas directement dans la plupart des propriétés de la matière condensée et peuvent donc être intégrés avec le noyau. Ne retenir que les électrons de valence n'entraîne donc pas de perte de précision et s'avère bien plus avantageux en temps de calcul. L'interaction entre les électrons et l'ensemble noyau-électrons de cœur est décrite par un potentiel effectif ou pseudopotentiel. Il en existe plusieurs types tels que ceux conservant la norme, l'ultra-soft (USPP) et le projecteur augmenté (PAW), ce dernier étant recommandé.

- Fonctionnelles: il en existe plusieurs familles comme LDA, GGA, non-locale et hybride (voir ci-dessus). Au sein de chaque famille, des fonctionnelles ont été spécialement optimisées pour différents types d'application (RPBE). Parmi les fonctionnelles couramment utilisées, nous pouvons citer la fonctionnelle GGA-PBE (Perdew-Burke-Enzerhoff).

- Magnétisme: la densité électronique en un point peut être séparée en deux contributions : celle du spin haut et celle du spin bas, chaque contribution étant déterminée en résolvant l'équation de 
Schroedinger dans laquelle $E_{x c}$ est spin-dépendante. Ces calculs concernent les méthodes LSDA mais peuvent être également appliqués à l'approximation GGA. Une description correcte de la structure magnétique peut augmenter directement ou indirectement la précision d'un calcul de phonon à travers la détermination de la structure d'équilibre correcte.

\section{Programmes :}

Le tableau suivant décrit de manière succinte quelques uns des programmes couramment utilisés pour le calcul de structures électroniques en physique de la matière condensée.

Tableau 1. Quelques programmes implémentant la TFD.

\begin{tabular}{|c|c|c|c|}
\hline Programme & $\begin{array}{l}\text { Méthode pour le } \\
\text { calcul de phonons }\end{array}$ & Base & Commentaires \\
\hline ABINIT [17] & Réponse linéaire & PW & \\
\hline CASTEP [18] & Réponse linéaire & différence finie & PW \\
\hline CPMD [19] & Réponse linéaire & différence finie & PW \\
\hline Quantum Expresso [20] & Réponse Linéaire & PW & \\
\hline VASP [21] & $\begin{array}{l}\text { Réponse linéaire } \\
\text { (version >5) \& } \\
\text { différence finie }\end{array}$ & PW & $\begin{array}{l}\text { Directement couplé } \\
\text { à PHONON, compatible } \\
\text { avec nMOLDYN }\end{array}$ \\
\hline WIEN2K [22] & Différence finie & Mélange & $\begin{array}{l}\text { Directement couplé } \\
\text { à PHONON }\end{array}$ \\
\hline DMOL3 [23] & Différence finie & Orbitales localisées & $\begin{array}{l}\text { Peut traiter les molécules, } \\
\text { groupes de molécules } \\
\text { et solides isolés }\end{array}$ \\
\hline GAUSSIAN [24] & $\begin{array}{l}\text { Vibrations moléculaires } \\
\text { avec différence finie et } \\
\text { dérivées analytiques }\end{array}$ & Orbitales localisées & $\begin{array}{l}\text { Le premier programme } \\
\text { de chimie quantique }\end{array}$ \\
\hline GAMESS [25] & $\begin{array}{l}\text { Vibrations moléculaires } \\
\text { avec différence finie } \\
\text { et dérivées analytiques }\end{array}$ & Orbitales localisées & $\begin{array}{l}\text { Une version académique } \\
\text { proche de Gaussian } \\
\text { SIESTA [15] }\end{array}$ \\
\hline Différence finie & Orbitales localisées & $\begin{array}{l}\text { Directement couple } \\
\text { à PHONON. } \\
\text { Méthode linéaire. }\end{array}$ & \\
\hline CRYSTAL [26] & Différence finie & Orbitales localisées & HF et non TFD \\
\hline
\end{tabular}

\section{Champ de force classiques}

Ces méthodes sont appelées classiques car elles ne considèrent pas explicitement les électrons et leurs aspects quantiques (e.g. fonction d'onde). Par exemple, un phénomène purement électronique telle que la formation de liaisons chimiques est représenté par des ressorts dont la longueur à l'équilibre et la raideur sont caractéristiques des atomes impliqués. Ce type de simplification permet aux méthodes $\mathrm{CF}$ d'être plusieurs ordres de grandeur plus rapides $(\sim 8)$ que les méthodes basées sur le calcul de la structure électronique.

Les champs de force de première génération comportent des ressorts pour décrire la longueur des liaisons, l'angle formée par trois atomes consécutifs et l'angle de torsion (dièdre) autour de la liaison centrale de quatre atomes consécutifs.

$$
E=\Sigma_{\text {bonds }} k_{i}^{\text {bond }}\left(d_{i}-d_{0}\right)^{2}+\Sigma_{\text {angles }} k_{i}^{\text {angle }}\left(\theta_{i}-\theta_{0}\right)^{2}+\Sigma_{\text {dihedrals }} k_{i}^{\text {dih }}\left[1+\cos \left(n \phi_{i}-\delta_{0}\right)\right]
$$


Les interactions inter-moléculaires son souvent décrites par le potentiel de Van der Waals (VDW),

$$
E_{V D W}=\Sigma_{i} \Sigma_{j \neq i} 4 \epsilon_{i j}\left|\left(\frac{\sigma_{i j}}{r_{i j}}\right)^{12}-\left(\frac{\sigma_{i j}}{r_{i j}}\right)^{6}\right|,
$$

et l'interaction coulombienne

$$
E_{\text {Coulomb }}=\Sigma_{i} \Sigma_{j \neq i} \frac{q_{i} q_{j}}{\varepsilon r_{i j}},
$$

qui dépend de la charge partielle $q$. La notion de liaison hydrogène peut également être prise en compte en ajoutant des interactions additionnelles sous forme de ressorts ou d'un potentiel de Coulomb avec des charges partielles modifiées.

Le temps de calcul d'une méthode CF étant directement lié au nombre de termes dans l'expression de l'énergie, les champs de force de première génération (CHARMM, AMBER ...) sont utilisés pour les systèmes biomoléculaires. Les champs de force de seconde et troisième génération comportent des termes additionnels tels que les termes croisés entre liaisons, angles et dièdres. Les paramètres d'un champ de force sont optimisés soit à partir de données expérimentales soit à partir de calculs $a b$ initio/TFD. La formulation simplifiée de l'énergie implique l'existence de paramètres spécifiques à des classes de molécules données. Par conséquent, l'utilisation de cette méthode impose quelques contraintes supplémentaires de la part de l'utilisateur comme le choix du champ de force le plus adapté, la définition de la topologie du système (connectivité, charges partielles ...). Contraintes qui n'existent pas ou peu lors d'un calcul ab initio/DFT.

Le gain en temps de calcul des méthodes CF par rapport aux méthodes basées sur la structure électronique a cependant un prix en termes de précision et de variété des propriétés physico-chimiques pouvant être modélisées. Au cours d'une simulation CF, une liaison chimique ne peut être rompue ou créée et les effets de polarisation dépendants du temps ne sont pas ou peu pris en compte (ces problèmes concernent plus particulièrement les systèmes moléculaires et ioniques où la polarisation est importante). Les modèles en couches où l'ion et le cortège électronique des atomes sont connectés par un ressort sont plus robustes [27]. Dans le cas des métaux, la méthode de l'atome intégré, dont les fondations reposent sur la TFD, est plus appropriée.

Codes :

Le tableau suivant décrit de manière succincte quelques uns des programmes couramment utilisés pour le calcul de type $\mathrm{CF}$ en physique de la matière condensée.

\section{DISCUSSION : COMBINER LES MÉTHODES CF ET TFD}

Une règle simple est d' "utiliser la TFD lorsque c'est possible". Les limites pratiques de la TFD imposent un nombre maximum d'atomes de quelques centaines pour un nombre maximum de calculs de l'ordre $10^{4}$. Lorsqu'un nombre inférieur de calculs est suffisant, comme par exemple dans le cas de dynamique sur réseau de systèmes à haut degré de symétrie, alors un nombre plus important d'atomes peut être envisagé. A l'ILL, la simulation de phonons de clathrates de Barium-Germanium comprenant 408 atomes dans la boîte de simulation a récemment été réalisée. Dans le cas de la DM, les $10^{4}$ calculs peuvent être effectués sur 200 atomes approximativement. Au-delà de ces limites, les méthodes CF prennent le relais sous réserve de la disponibilité des paramètres pour le système étudié.

Une autre limitation des méthodes TFD qui doit être soulignée réside dans l'étude de matériaux amorphes ou partiellement cristallins [36]. Pour ces matériaux, le bruit résultant de la résolution numérique de l'équation de Schroedinger peut devenir comparable aux différences d'énergies entre des minima locaux de la SEP. Par conséquent, il peut être difficile en pratique de déterminer de manière correcte un minimum de la SEP et les grands déplacements $(\sim 0.05 \AA)$ requis par la méthode TFD peuvent entraîner la divergence du système par rapport à sa structure d'équilibre (aboutissant à des 
Tableau 2. Quelques programmes implémentant les méthodes classiques.

\begin{tabular}{|c|c|c|}
\hline Programme & $\begin{array}{l}\text { Trajectoires compatibles } \\
\text { avec nMOLDYN }\end{array}$ & Commentaires \\
\hline GULP [27] & & $\begin{array}{l}\text { Programme pour le calcul de } \\
\text { dynamique sur réseau, version } \\
\text { gratuite disponible. Version } \\
\text { commerciale interfacée } \\
\text { avec Materials Studio }\end{array}$ \\
\hline Discover [28] & $\mathrm{Y}$ & $\begin{array}{l}\text { Version commerciale disponible } \\
\text { dans Materials Studio [29] }\end{array}$ \\
\hline DL_POLY [30] & Y & \\
\hline LAMMPS [31] & & $\begin{array}{l}\text { Programme académique. } \\
\text { Version commerciale interfacée } \\
\text { avec MAPS [32] }\end{array}$ \\
\hline NAMD [33] & $\mathrm{Y}$ & Couramment utilisés pour \\
\hline CHARMM [34] & Y & la DM de systèmes biomoléculaires. \\
\hline AMBER [35] & $\mathrm{Y}$ & Champ de force de première génération. \\
\hline
\end{tabular}

fréquences imaginaires dans un calcul type DR). En comparaison, les méthodes CF sont analytiques et la structure à l'équilibre peut donc être déterminée avec des forces résiduelles plusieurs ordres de grandeur plus petites que celles obtenues avec les méthodes TFD $\left(10^{-8}\right.$ comparé à $\left.10^{-3} \mathrm{eV} / \AA ̊ \AA\right) . \mathrm{Si}$ des dérivations numériques sont utilisées à la place des dérivations analytiques pour le calcul des forces inter-atomiques, ces forces résiduelles ne sont plus que 2-3 ordres de grandeur plus petites $\left(10^{-4} \mathrm{eV} / \AA\right)$.

Cependant, si un champ de force approprié existe, il peut être intéressant de combiner les méthodes CF et TFD.

\section{Exemple :}

Lors d'une étude récente sur les conducteurs de l'ion oxygène, les phonons d'une structure de millérite $\left(\mathrm{Ca}_{2} \mathrm{Fe}_{2} \mathrm{O}_{5}\right)$ ont été calculés en TFD donnant un très bon accord avec le GDEV expérimental [37]. Le materiau $\mathrm{Sr}_{2} \mathrm{Fe}_{2} \mathrm{O}_{5}$, montre une symétrie crystalline plus élevée liée au désordre des positions d'atomes d'oxygènes dans des couches de tetraèdre $\mathrm{Fe}_{4} \mathrm{O}_{4}$ (les couches d'octaèdre $\mathrm{Fe}-\mathrm{O}_{6}$ étant ordonnée) et les calculs de DR ne sont pas envisageables. Comprendre la différence entre le $\mathrm{Ca}$ et le $\mathrm{Sr}$ est de première importance dans la mesure où le désordre au niveau du réseau de l'oxygène entraîne une plus grande mobilité de l'oxygène et ceci particulièrement à des températures proches de la température ambiante. D'un point de vue structural, le principal effet de la présence de l'ion Sr est d'augmenter le plus grand des paramètres de la maille cristalline ce qui étire l'octaèdre Fe-O6. Le modèle en couche de Bush [38], implémenté dans GULP, a donné un bon accord avec les calculs de phonons en TFD avec pour principale différence un temps de calcul de quelques minutes avec GULP là où le calcul TFD a duré plusieurs jours. La méthode CF a ensuite été utilisée pour calculer les phonons en fonction du grand axe de la maille cristalline. Ce paramètre étant augmenté dans le cas du $\mathrm{Ca}$ et diminué dans le cas du Sr. Les résultats de cette étude sont présentés dans la Figure 12. Un phonon de $\mathrm{Ca}_{2} \mathrm{Fe}_{2} \mathrm{O}_{5}$ avec une fréquence de $10 \mathrm{meV}$ pour la maille cristalline optimisée baisse avec l'augmentation du paramètre de maille le plus long et le mode devient imaginaire au dela d'un paramètre de maille de $15.1 \AA$. Dans le cas des molécules avec le Sr, diminuer le paramètre de maille en dessous de $15.1 \AA ̊$ donne un mode stable. Ceci montre clairement le rôle critique joué par ce paramètre de maille par rapport à la nature de l'atome. Le mode en question est polarisé le long du grand axe de la maille et à tendance à séparer l'oxygène apical de l'octaèdre Fe-O6. Un autre exemple de complémentarité entre méthodes classiques et TFD est fourni par des simulations DFT MD de la molécule avec le Sr à $600 \mathrm{~K}$. Celles-ci ont montré une certaine instabilité lors de la migration de l'oxygène apical de l'octaèdre vers les couches tétraédriques. A la même température, le réseau des oxygènes des molécules avec le Ca est stable. 


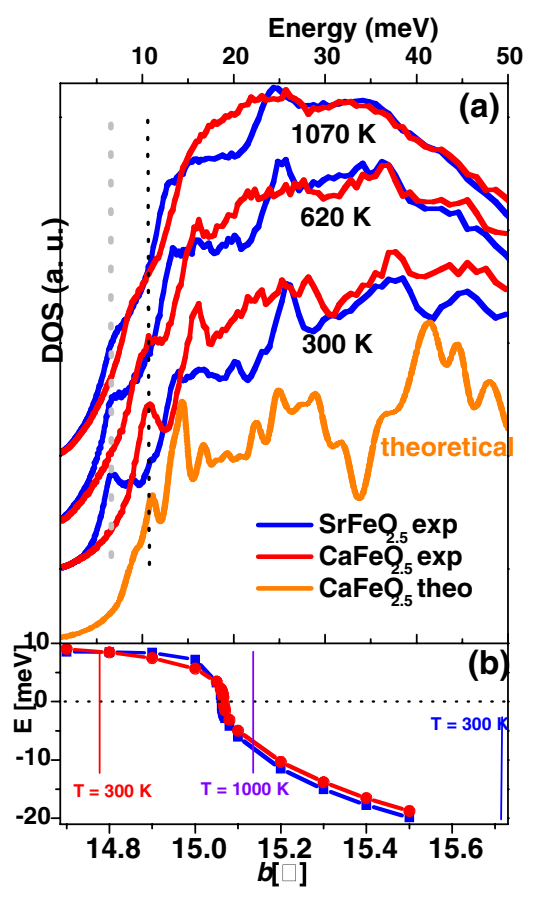

Figure 12. GDEV extrait de données DIN pour le $\mathrm{Ca}_{2} \mathrm{Fe}_{2} \mathrm{O}_{5}$ (rouge) et le $\mathrm{Sr} 2 \mathrm{Fe} 2 \mathrm{O} 5$ (bleu) comparé avec des calculs TFD DR réalisés sur le $\mathrm{Ca}_{2} \mathrm{Fe}_{2} \mathrm{O}_{5}$. Des simulations CF utilisant un modèle en couche (réalisées avec GULP) donnent des résultats comparables, permettant de réaliser rapidement 30 simulations CF DR sur deux systèmes pour montrer l'effet du paramètre de maille $\mathrm{b}$ sur le phonon à $10 \mathrm{meV}$ responsable de la conduction de l'ion oxygène dans le $\mathrm{Sr}_{2} \mathrm{Fe}_{2} \mathrm{O}_{5}$ à des températures juste au-dessus de la température ambiante.

\section{CONCLUSION}

Dans le contexte de la DIN, nous avons présenté deux méthodes, la méthode DR et la méthode DM, pour le calcul de $S(Q, \omega)$ et d'autres grandeurs associées. Ces méthodes sont basées sur des calculs d'énergie totale à partir des méthodes TFD et CF. Les quatre combinaisons sont possibles, le tout dépendant du contexte d'utilisation. La DR est typiquement appliquée aux systèmes avec plus de symétrie et moins d'atomes car dans ces cas là, la dispersion des modes de vibrations peut être étudiée en détail par les voies expérimentale et numérique. Les méthodes TFD peuvent être appliquées, et sont d'ailleurs essentielles, lorsqu'il s'agit de traiter en détail la structure électronique de matériaux composés d'atomes riches en électrons. Les méthodes CF sont en général plutôt réservées à des systèmes de taille plus importante non accessibles actuellement aux méthodes TFD. Pour ces systèmes, complexes par essence, les relations de dispersion au niveau atomique ne sont en général pas d'un intérêt fondamental et la DM est alors utilisée pour l'obtention de grandeurs liées à la dynamique du système. Pour résumer, les approches couramment utilisées pour l'analyse de données issues de la diffusion inélastique de neutrons sont $« \mathrm{DR}+\mathrm{TFD} »$ et $\ll \mathrm{DM}+\mathrm{CF} »$.

La TFD offre une grande précision sur un grand intervalle de fréquences avec peut-être une précision moindre pour les basses fréquences où les incertitudes sur les solutions numériques de l'équation de Schroedinger ne sont plus négligeables. Les méthodes $\mathrm{CF}$, quant à elles, peuvent être assez précises à basses fréquences dans la mesure où (a) ces méthodes sont analytiques et (b) les modes collectifs dépendent de la valeur moyenne d'un grand nombre de constantes de forces inter-atomiques. Par contre, le manque de précision sur des constantes de force spécifiques à hautes fréquences peut devenir préjudiciable. De manière générale, les méthodes $\mathrm{CF}$ offrent moins de précision que les méthodes TFD. 
Dès lors qu'un bon accord a été obtenu entre l'expérience et la simulation, les résultats numériques doivent être analysés. Etant donné le nombre relativement limité d'atomes considérés lors d'un calcul TFD, les résultats peuvent être analysés atome par atome. Pour des simulations de type CF, la taille des systèmes est souvent plus importante ce qui impose une analyse par groupe d'atomes, par exemple, les nucléotides de l'ADN [3] ou les acides aminés des protéines.

Enfin, dans le cas des poudres ou des expériences en phase liquide, grâce aux derniers développements effectués dans le programme de simulation d'instrument McStas [39], il est désormais possible de réaliser des expériences virtuelles complètes. Dans ce programme, les neutrons traversent des instruments optiques (e.g. guides,monochromateurs etc), pour être finalement diffusés au niveau de l'échantillon. La probabilité de diffusion en fonction du transfert d'énergie et de la quantité de mouvement est déterminée par le calcul de $S(Q, \omega)$ et la probabilité qu'un évènement de diffusion ait lieu est reliée à la longueur du chemin parcouru par le neutron dans l'échantillon. Il est également possible dans ces expériences virtuelles d'accéder en détail aux phénomènes d'auto-écrantage et de diffusion multiple [40]. De plus, il est possible de simuler des artefacts dans la $S(Q, \omega)$ mesuré provenant de la diffusion parasite entre l'échantillon et son environnement (e.g. cryoaimant).

\section{Références}

[1] Parlinski, K., Am. Inst. Phys. Conference Proceedings 479, 121 (1999)

[2] Merzel, F. et al, Comp. Phys. Comm., 177, 530 (2007)

[3] Merzel, F. et al., Phys. Rev. E, 76, 31917 (2007)

[4] Plazanet, M. et al., J. Am. Chem. Soc., 127, 6672 (2005)

[5] Plazanet, M. et al., J. Chem. Phys., 115, 3241 (2001)

[6] Zbiri, M. et al., http://adsabs.harvard.edu/abs/2008arXiv0810.3941Z

[7] Baroni, S. et al., Rev. Mod. Phys., 73, 515-562 (2001).

[8] Johnson, M. R. et al., Nucl. Inst. Methods A, 600, 226 (2009).

[9] Koza, M. et al., Nature Materials, 7, 805 (2008)

[10] http://dirac.cnrs-orleans.fr/plone/software/nmoldyn/, Rog, T. et al., J. Comp. Chem. 24, 657 (2003).

[11] Zaharko, O. et al., Phys. Rev. B, 77, 224408 (2008)

[12] Brooks, B. R. et al. J. Comp. Chem., 16, 1522 (1994).

[13] Fontaine-Vive, F. et al., J. Chem. Phys., 124, 234503 (2006)

[14] Kohn, W., Phys. Rev. Letts., 76, 3168 (1996)

[15] http://www.uam.es/departamentos/ciencias/fismateriac/siesta/

[16] http://accelrys.com/products/materials-studio/modules/onetep.html

[17] http://www.abinit.org/

[18] http://www.tcm.phy.cam.ac.uk/castep/index.html

[19] http://www.cpmd.org/

[20] http://www.quantum-espresso.org/

[21] http://cms.mpi.univie.ac.at/vasp/

[22] http://www.wien2k.at/

[23] http://accelrys.com/products/materials-studio/modules/dmol3.html

[24] http://www.gaussian.com/

[25] http://www.cfs.dl.ac.uk/

[26] http://www.crystal.unito.it/

[27] https://www.ivec.org/gulp/

[28] http://accelrys.com/products/materials-studio/modules/discover.html

[29] http://accelrys.com/products/materials-studio/

[30] http://www.cse.scitech.ac.uk/ccg/software/DL_POLY/

[31] http://lammps.sandia.gov/ 
[32] http://www.scienomics.com/products/maps_platform.php

[33] http://www.ks.uiuc.edu/Research/namd/

[34] http://www.charmm.org/

[35] http://ambermd.org/

[36] Fontaine-Vive, F. et al., Chem. Phys., 124, 234503 (2009)

[37] Paulus, W. et al., J. Am. Chem. Soc., 130, 16080 (2008)

[38] Bush, T. S. et al., J. Mater. Chem.,4, 831 (1994).

[39] http://neutron.risoe.dk/

[40] Hugouvieux, V. et al., Physica B, 350, E151 (2004) 